\title{
Fluorometric assay for quantitation of biotin covalently attached to proteins and nucleic acids
}

\author{
Robert H. Batchelor, Adam Sarkez, W. Gregory Cox, and lain Johnson \\ Molecular Probes Detection Technologies, Invitrogen, Eugene, OR, USA \\ BioTechniques 43:503-507 (October 2007) \\ doi 10.2144/000112564
}

\begin{abstract}
As a component of the (strept)avidin affinity system, biotin is often covalently linked to proteins or nucleic acids. We describe here a microplate-based high-throughput fluorometric assay for biotin linked to either proteins or nucleic acids based on fluorescence resonance energy transfer (FRET). This assay utilizes a complex of Alexa Fluor ${ }^{\circledR} 488$ dye-labeled avidin with a quencher dye, 2-(4'-hydroxyazobenzene) benzoic acid (HABA), occupying the biotin binding sites of the avidin. In the absence of biotin, HABA quenches the fluorescence emission of the Alexa Fluor 488 dyes via FRET. HABA is displaced when biotin binds to the Alexa Fluor 488 dye-labeled avidin, resulting in decreased FRET efficiency. This mechanism results in an increase in fluorescence intensity directly related to the amount of biotin present in the sample. The assay is able to detect as little as 4 pmol biotin in a $0.1 \mathrm{~mL}$ volume within 15 min of adding sample to the reagent, with a Z-factor $>0.9$.
\end{abstract}

\section{INTRODUCTION}

The affinity of the proteins streptavidin and avidin for the small molecule biotin $\left(K_{d}\right.$ approximately $\left.10^{-15} \mathrm{M}\right)$ is one of the strongest noncovalent interactions known (1). The extremely high affinity makes this interaction a useful tool; proteins and nucleic acids labeled with biotin are the basis of a large number of biological detection systems (1). A key parameter that affects the performance of many of these systems is the number of attached biotins per macromolecule (degree of labeling or DOL). However, biotin is difficult to detect and quantitate directly because its intrinsic absorption is difficult to distinguish from that of proteins and nucleic acids.

Many assays have been developed to work around this problem. One of the first assays developed was the absorbance-based 2-(4'-hydroxyazobenzene) benzoic acid (HABA) assay developed by Green (2). HABA binds with moderate affinity to the biotin binding site of avidin. In the presence of biotin, HABA is displaced, and its absorption maximum shifts from 500 to $350 \mathrm{~nm}$. The amount of biotin is determined by measuring the decrease of absorbance at $500 \mathrm{~nm}$. This assay has a detection limit of 200 pmol biotin. Another assay was developed by Huang et al. in the 1990s (3). Based on the competition of free biotin for binding sites of soluble avidin- $\beta$-galactosidase, the assay has a sensitivity of approximately $100 \mathrm{pmol}$ but requires washing steps for maximal sensitivity. Another assay for biotin compound quantitation was described by Dorris et al. (4). This assay is more sensitive than the HABA assay-60 pmol-but requires the use of highperformance liquid chromatography (HPLC). Another assay that is more amenable to high-throughput analysis is a colorimetric competition assay developed by Gan and Marquardt (5) that can detect as little as $1.6 \mathrm{fmol}$ biotin. However, like the Huang assay, it requires a wash step. More recently, $\mathrm{Wu}$ et al. developed a kinetic assay based on the fluorescence enhancement of fluorescein biotin when it is displaced from avidin (6). While this assay has an excellent limit of sensitivity (approximately $150 \mathrm{fmol}$ ), low concentrations of biotin require more than $1 \mathrm{~h}$ to achieve equilibrium. While useful, these assays all lack at least one of the following four operationally desired characteristics: no-wash, high-throughput, rapid readout, and sensitivity.

Here we describe a fluorogenic assay for biotin that addresses all four of these issues. This assay is based on fluorescence resonance energy transfer (FRET) quenching and dequenching (Figure 1) of the Biotective ${ }^{\mathrm{TM}}$ Green reagent. The Biotective Green reagent consists of avidin covalently labeled with the fluorescent dye Alexa Fluor ${ }^{\circledR}$ 488 and complexed with a quencher dye, HABA, occupying the biotin binding sites of the avidin. In the absence of biotin, HABA quenches the fluorescence of the Biotective Green reagent via FRET. When biotin or one of its compounds (biotin covalently linked to another small molecule) binds to the Biotective Green reagent, HABA is displaced from the avidin, eliminating the quenching of the fluorescent Alexa Fluor 488 dye. The FRET mechanism results in an increase in fluorescence corresponding to the amount of biotin or biotin compound present in the sample. After nuclease or protease pretreatment of samples, the assay is complete within $5 \mathrm{~min}$ without washing, can detect as little as 4 pmol biotin in $0.1 \mathrm{~mL}$ volume, and is amenable to high-throughput assay formats.

\section{MATERIALS AND METHODS}

5-(biotinylaminohexylacrylamido)dUTP (biotin-aha-dUTP), 5-(biotinylaminohexylacrylamido)-dUMP (biotin-aha-dUMP), and 5-(biotinylaminohexylacrylamido)-uridine (biotin-aha-uridine) were obtained from Berry \& Associates (Dexter, MI, USA). Biotective Green is commercially available from Molecular Probes Detection Technologies, Invitrogen (Eugene, OR, USA) as a component of the FluoReporter ${ }^{\circledR}$ Biotin Quantitation Assay kit. Biotin, biocytin ( $\varepsilon$-biotinoylL-lysine), 6-((6-((biotinoyl)amino) hexanoyl)amino)hexanoic acid (biotin-XX), phosphate-buffered saline (PBS), and goat anti-mouse immunoglobulin $\mathrm{G}(\mathrm{IgG})$ labeled with an average of 5.5 biotin-XX moieties were all obtained from Molecular Probes Detection Technologies, Invitrogen. HPLC-purified hexabioti- 
nylated oligonucleotide was purchased from Qiagen (Valencia, CA, USA), sequence $\mathrm{C}[$ biotin $\sim \mathrm{dT}] \mathrm{CATAGC}$ [biotin dT]CACGC[biotin dT]GTAGG [biotin dT]ATC[biotin dT]CAG[biotin dT]TCGG, where [biotin dT] is biotinylated deoxythymidine. Sodium borate, sodium hydroxide, calcium chloride, and Pronase E were obtained from Sigma-Aldrich (St. Louis, MO, USA). Micrococcal nuclease was from Worthington Biochemical (Lakewood, NJ, USA). The plates used were clear round-bottomed 96-well plates from Corning Costar (Lowell, MA, USA).

The Biotective Green reagent was reconstituted to a $2 \times$ working concentration by adding $1.75 \mathrm{~mL} 1 \times$ PBS buffer to the vial supplied by the vendor. The final concentration in the well was $200 \mathrm{nM}$ Alexa Fluor 488avidin and $250 \mu \mathrm{M}$ HABA in $1 \times$ PBS. The standards (either biotin or biotin compounds) were diluted in the same buffer to a final concentration of 3 or 4 $\mu \mathrm{M}$ and serially diluted in $50 \mu \mathrm{L}$ buffer in a microplate well. An equal volume $(50 \mu \mathrm{L})$ of $2 \times$ Biotective Green reagent was added to all wells containing either sample or standards. The resulting fluorescence intensities were measured in a CytoFluor 4000 fluorescent microplate reader (PerSeptive Biosytems, Framingham, MA, USA) within $15 \mathrm{~min}$ of Biotective Green reagent addition. Fluorescence emission was measured from the bottom of the plate at $530 \mathrm{~nm}$ $\pm 12.5 \mathrm{~nm}$, using excitation of $485 \mathrm{~nm}$ $\pm 10 \mathrm{~nm}$.

The biotin compounds biotin-ahadUTP, biotin-aha-dUMP, biotin-ahauridine, biocytin, and biotin-XX-free acid were weighed out and resuspended in distilled water. Biotin was weighed out and resuspended in $0.1 \mathrm{M}$ sodium hydroxide.

To determine the amount of biotin from a biotinylated nucleic acid, a hexabiotinylated oligonucleotide standard was digested overnight in 50 $\mathrm{mM}$ borate, $\mathrm{pH} 9.3,2 \mathrm{mM} \mathrm{CaCl}_{2}$, at $37^{\circ} \mathrm{C}$ with micrococcal nuclease at a final concentration of $40 \mathrm{U} / \mathrm{mL}(1 \mathrm{U}$ is defined as the amount required to cause a change in optical density of 1.0 at $260 \mathrm{~nm}$ at $37^{\circ} \mathrm{C}, \mathrm{pH} 8.0$, using DNA as the substrate). The resulting digest was diluted in PBS and mixed with the Biotective Green reagent as described

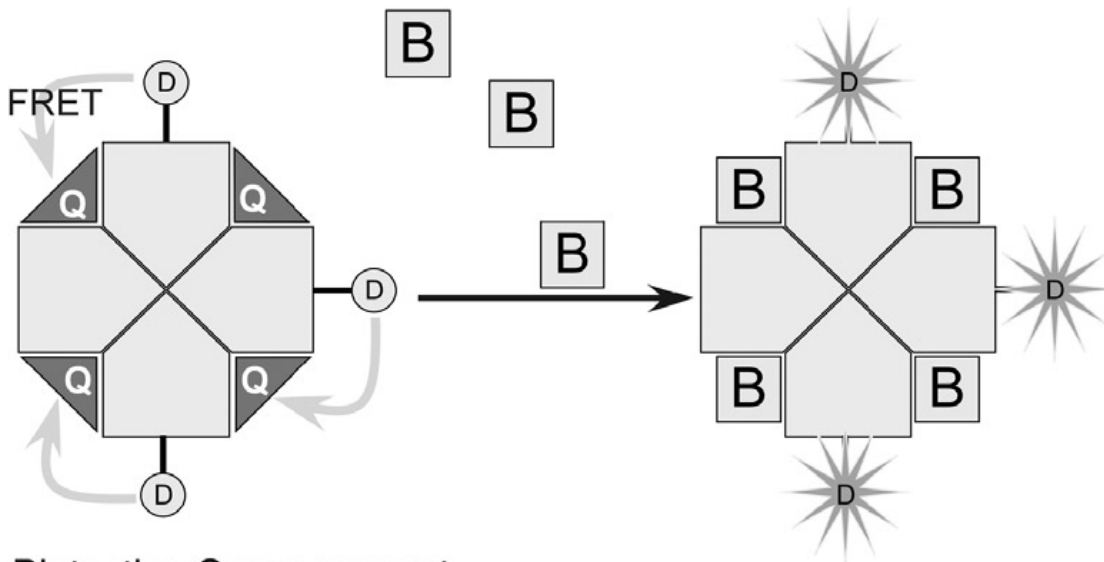

\section{Biotective Green reagent}

Figure 1. Response mechanism of the Biotective Green reagent-based assay. Before biotin or a biotin compound (indicated as B) is added to the Biotective Green reagent, the fluorescence of the Alexa Fluor 488 dye (indicated as $\mathrm{D}$ ) is quenched by fluorescence resonance energy transfer (FRET) to the quencher dye 2-(4'-hydroxyazobenzene) benzoic acid HABA (indicated as Q). After biotin is added to the reaction mix, the HABA is displaced according to the amount of biotin present, resulting in a corresponding increase in fluorescence.

Table 1. Calculation of Biotin DOL

\begin{tabular}{|c|c|c|c|c|}
\hline \multirow{2}{*}{$\begin{array}{l}6 \text { Biotin-Oligonucleotide (DOL 6.0) } \\
\text { Actual Biotina } \\
\text { (pmol) }\end{array}$} & \multicolumn{2}{|c|}{ Digested } & \multicolumn{2}{|c|}{ Undigested } \\
\hline & Calculated & DOLc & Calculated & DOL \\
\hline 60 & 47.5 & 4.75 & 30 & 3 \\
\hline 30 & 29.5 & 5.9 & 15.5 & 3.1 \\
\hline 15 & 14.5 & 5.8 & 8.9 & 3.56 \\
\hline 7.5 & 8.6 & 6.88 & 4.4 & 3.52 \\
\hline \multirow{2}{*}{$\begin{array}{l}\text { Biotin-XX-IgG (DOL 5.5) } \\
\text { Actual Biotin } \\
\text { (pmol) }\end{array}$} & \multicolumn{2}{|c|}{ Digested } & \multicolumn{2}{|c|}{ Undigested } \\
\hline & Calculated & DOL & Calculated & DOL \\
\hline 68.75 & 53 & 4.24 & 39 & 3.12 \\
\hline 34.38 & 29 & 4.64 & 23 & 3.68 \\
\hline 17.19 & 14 & 4.48 & 13 & 4.16 \\
\hline 8.59 & 7 & 4.48 & 7 & 4.48 \\
\hline \multicolumn{5}{|c|}{$\begin{array}{l}\text { Relative calculated biotin in digested and undigested biotin from } 6-((6-((\text { biotinoyl)amino) } \\
\text { hexanoyl)amino)hexanoic acid immunoglobulin } G \text { (biotin-XX-lgG) and biotinylated oligonucleotide. } \\
\text { Both biotin degree of labeling (DOL) of both the biotin-XX-lgG and biotinylated oligonucleotide were } \\
\text { determined by matrix-assisted laser desorption/ionization time-of-flight (MALDI-TOF) mass spectrom- } \\
\text { etry (data not shown). The standard for the hexabiotin oligonucleotide was 5-(biotinylaminohexylacryl- } \\
\text { amido)-dUMP (biotin-aha-dUMP). The standard used for the biotin-XX-lgG was biocytin. } \\
\text { aThe moles of conjugate analyzed multiplied by the known DOL. } \\
\text { bThe moles of biotin determined using the Biotective Green reagent. } \\
\text { cDegree of labeling calculated from the Biotective Green assay data. }\end{array}$} \\
\hline
\end{tabular}

previously. We confirmed the molecular weight of the hexabiotinylated oligonucleotide via matrix-assisted laser desorption/ionization time-of-flight (MALDI-TOF) mass spectrometry (data not shown).

To determine the amount of biotin from a biotinylated protein, a biotin-
XX-goat anti-mouse IgG standard was obtained, and the degree-of-biotinylation determined by MALDI-TOF mass spectrometry (data not shown). This protein was digested overnight in $1 \times$ PBS buffer at $37^{\circ} \mathrm{C}$ with the protease Pronase $\mathrm{E}$ at a final concentration of $14.3 \mathrm{U} / \mathrm{mL}$ [1 U is defined 


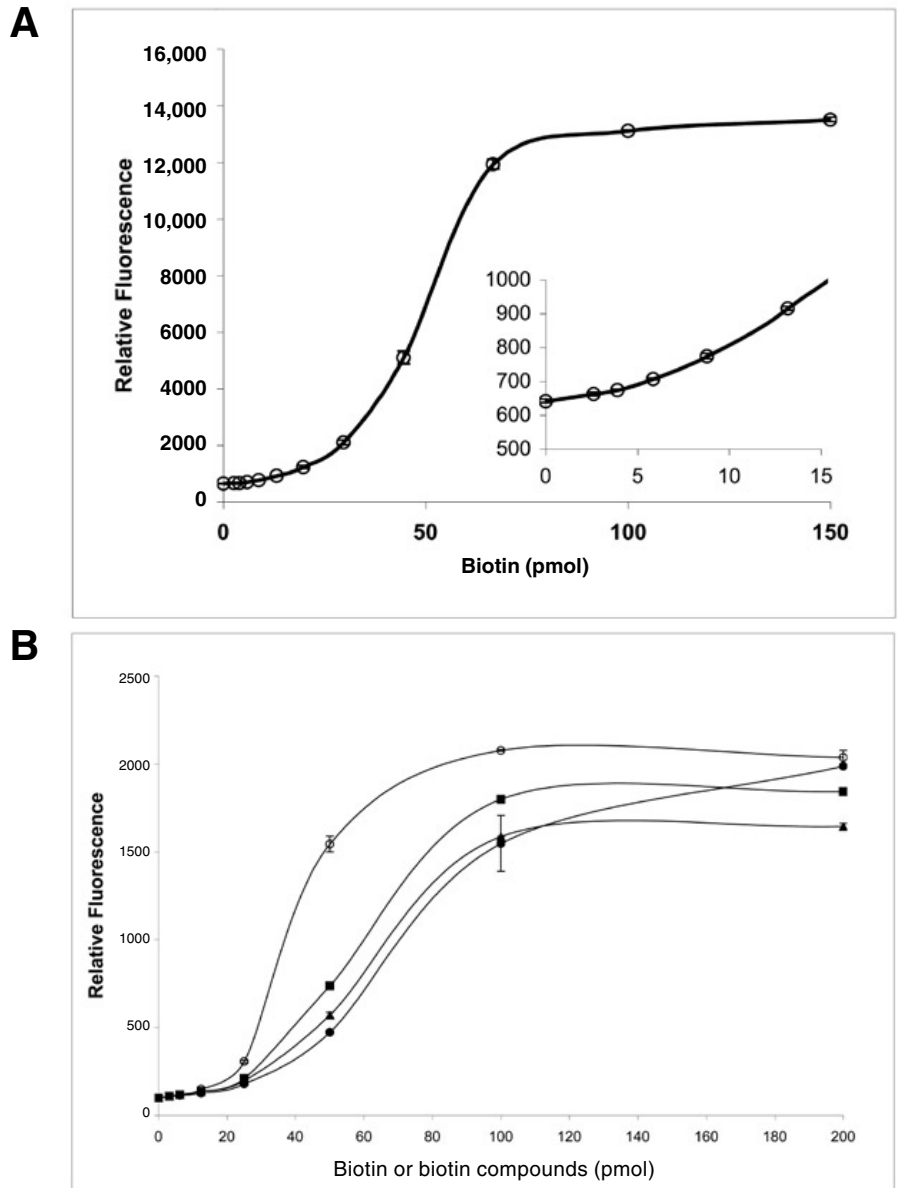

Figure 2. Relative fluorescence of biotin and biotin compounds in the presence of Biotective Green reagent. (A) Relative fluorescence signal of biotin titration in a 96-well plate. Biotin was serially diluted across a 96-well plate, Biotective Green reagent was added, and the resulting fluorescence signal was determined as per the Materials and Methods section. Error bars are one standard deviation of the mean of three separate wells and are within the symbols used to represent the data points. Inset shows the lower concentrations of the same experiment for clarity. (B) Relative fluorescent signal of biotin compared with various biotin compounds. Biotin (O), biocytin (๑), 5-(biotinylaminohexylacrylamido)-dUMP (biotin-aha-dUMP; $\boldsymbol{\square}$ ), or biotin-aha-dUTP 5-(biotinylaminohexylacrylamido)-dUTP; $\boldsymbol{\Delta}$ ) were serially diluted across a 96well plate, Biotective Green reagent was added, and the resulting fluorescence signal was determined as per the Materials and Methods section. Error bars are one standard deviation of the mean of three separate wells. Inset shows the lower concentrations of the same experiment for clarity.

as the amount required to hydrolyze casein to produce color equivalent to $1.0 \mu \mathrm{mol}$ tyrosine per minute at $\mathrm{pH}$ 7.5 at $37^{\circ} \mathrm{C}$ (color by Folin-Ciocalteu reagent)]. The resulting digestion mixture was diluted in PBS and mixed with the Biotective Green reagent as described previously. Separate experiments demonstrated that the Pronase E did not impact the fluorescence of the assay (via digestion of the Biotective Green reagent) within the timeframe of the assay (data not shown).

For both biotinylated macromolecules, a standard curve of either biocytin or biotin-aha-dUMP, for protein or nucleic acids, respectively, was run within the same assay as the digested biotinylated macromolecules. Biotin concentrations corresponding to the fluorescence intensities of unknown samples were obtained by linear interpolation between the standard intensity values immediately above and below that of the unknown.

\section{RESULTS AND DISCUSSION}

Figure 1 illustrates the mechanism of the Biotective Green-based detection system. The combination of three factors - spectral overlap, size of avidin, and biotin displacement of the quencher-are the basis of the Biotective Green reagent-based assay.
When bound to avidin, the absorption spectrum of HABA substantially overlaps the fluorescence emission spectrum of the Alexa Fluor 488 dye (data not shown). The calculated Förster radius $\left(R_{0}\right)$ of the Alexa Fluor 488 dye/HABA donor/acceptor pair is $5.6 \mathrm{~nm}$. Thus efficient FRET is possible across the entire avidin tetramer [dimensions $5.6 \times 5.0 \times 4.0$ $\mathrm{nm}$ (7)]. Furthermore, the working concentration of HABA $(250 \mu \mathrm{M})$ used to form the Biotective Green complex is designed to produce nearly complete saturation of biotin binding sites [97.6\% saturation calculated based on $K_{d}=6 \mu \mathrm{M}$ for HABA binding to avidin (8)]. Accordingly, quenching of Alexa Fluor 488 fluorescence in the intact complex is highly efficient [ $>95 \%$ calculated as $100^{*}$ $\left(1-\left(F_{d a} / F_{d}\right)\right)$ where $F_{d a}$ and $F_{d}$ are the fluorescence intensities at the beginning and end points, respectively, of the biotin titration shown in Figure 2A]. As in the spectrophotometric HABA assay (2), the much lower avidin-binding affinity of HABA results in efficient displacement by biotin. However detection of the displacement by FRET affords an increase in sensitivity of more than 100-fold. Displacement of HABA by biotin results in decreased FRET due to decreased donor/acceptor proximity and also due to decreased spectral overlap between the Alexa Fluor 488 dye and HABA.

To illustrate the response of the reagent to free biotin, Figure $2 \mathrm{~A}$ demonstrates the change in fluorescence over a biotin titration; increasing amounts of biotin result in increasing fluorescence. Having determined the responsiveness of the Biotective Green reagent to biotin, our primary aim was to determine the moles of biotin per mole of protein or DNA, also known as degree of labeling (DOL), of biotinylated nucleic acids and proteins. When we first attempted to determine the biotin DOL of our standards the calculated DOL results were consistently below the expected DOL. This was not unexpected, as previous reports in the literature $(9,10)$ have underestimated biotin DOL of macromolecules due to steric hindrance of access to avidin-binding sites. 
The steric hindrance of a biotin attached to a protein or DNA molecule could effectively decrease the affinity of the biotin for the Biotective Green reagent. Alternatively, multiple biotins could be attached very close to one another on the macromolecule. This could arise during either chemical modification, such as biotin succinimidyl ester labeling of lysines on a protein, or through incorporation of biotin compounds during synthesis, such as incorporation of biotin-dUTP into an oligonucleotide. Regardless of the labeling mechanism, biotins close to each other would inevitably result in at least one cryptic or masked biotin. Cryptic biotins will not be detected by the Biotective Green reagent as the avidin component can only resolve biotins that are further apart than the four biotin binding sites on avidin, a few nanometers (7). Thus, the total amount of biotinylation of molecules with a high-biotin DOL will be underestimated by the Biotective Green reagent unless the biotinylated molecule is digested. However, it should be noted that many assays that utilize the biotin(strept)avidin interaction are based on the number of (strept)avidins that bind to biotin (1). These assays report the effective biotin amount, not the total. The effective biotin concentration is the total biotin concentration minus the cryptic and sterically hindered biotins described previously. In order to determine the total amount of biotinylation, we used micrococcal nuclease to digest biotinylated nucleic acids and the protease Pronase E to digest biotinylated proteins, similar to Rao et al. (9). The total biotinylation of both the oligonucleotide and goat anti-mouse $\operatorname{IgG}$ were also determined via MALDI-TOF mass spectrometry (data not shown). For the data in Table 1 , the calculated amount of unknown biotin in the assay was compared with the expected amount of biotin based on the known amount of biotinylation from MALDI-TOF mass spectrometry of the biotinylated macromolecules. Table 1 demonstrates the differences in fluorogenic response when a biotinylated macromolecule (protein or DNA) sample is added to a solution of Biotective Green reagent with or without prior digestion. The average of the dilution series of the digested hexabiotinylated oligonucleotide is $<3 \%$ off from the expected amount of biotin. The same calculation for the undigested hexabiotinylated oligonucleotide is approximately $45 \%$ less than expected, demonstrating that there are approximately three cryptic biotins in this oligonucleotide that are revealed by digestion. The average of the dilution series of the digested biotin-XX-IgG is approximately $20 \%$ less than expected. The difference between the DOL of the digested biotin-XX-IgG and the calculated DOL may be due to slight error in both the MALDI-TOF mass spectrometry and the assay. The average of the dilution series of the undigested biotin-XX-IgG is approximately $30 \%$ less than expected. Overall, Table 1 demonstrates that digested macromolecules result in more accurate determination of the total biotin compound present within the well. The end user of the Biotective Green reagent, or any other assay that quantifies the amount of biotin via a (strept)avidin-based mechanism, should consider if they need to determine either the effective or total biotin within a sample.

Even after adding a digestion step to unmask any cryptic or sterically hindered biotins, the calculated biotin DOL of protein and nucleic acids were still below the expected DOL. This can be partly attributed to biotin derivatives having lower affinities for the Biotective Green reagent than that of free biotin alone. The biotin derivatives standard curves shown in Figure 2B demonstrate that equal concentrations of different biotin derivatives results in varying amounts of fluorogenic response when combined with the Biotective Green reagent in solution. Presumably this is due to differing affinities of the biotin derivatives for avidin, one of the components of the Biotective Green reagent. Regardless of mechanism, the biotin compounds clearly have differences in ability to displace the quencher dye, HABA, and hence differing abilities to relieve the FRET quenching. This leads to varying amounts of fluorogenic response.

This difference in fluorescence results in significant differences in the amount of biotin contained within a sample that is estimated from the standard curve.

Thus, if one used biotin as the standard for the assay when measuring the DOL of biotinylated DNA, for example, the assay would substantially underestimate the total amount of biotin in the sample, as biotin generates more fluorescence than equimolar biotinaha-dUTP (Figure 2B). To correct for this, we used biotin compounds as standards that more closely match the biotin compounds that are generated upon digestion of biotinylated macromolecules. For proteins we use biocytin ( $\varepsilon$-biotinoyl-L-lysine), and for nucleic acids we use biotin-aha-dUMP (Figure 1). Equal amounts of biotin-aha-UMP and biotin-aha-dUMP generate the same fluorogenic response in solution with the Biotective Green reagent (data not shown). The lower limit of detection within the assay utilizing either biocytin or biotin-aha-dUMP is about 4 pmol in $0.1 \mathrm{~mL}$ (Figure 2B, inset), where the lower limit of detection is defined as the lowest amount of analyte distinguishable above three standard deviations above background. The $Z$-factor-a dimensionless measure of an assay's signal window (11) —of the assay is $>0.9$ (data not shown) for both biotin compounds. In terms of the absolute amount of biotin detected, the lower limit of detection can be reduced by simply using smaller samples volumes. Using a NanoDrop ${ }^{\circledR}$ ND-3300 fluorometer (NanoDrop Technologies, Wilmington, DE, USA), a lower limit of detection of 0.04 pmol biocytin in 2 $\mu \mathrm{L}$ was obtained (data not shown).

In summary, here we describe a fluorometric assay for the quantitation of biotin and its derivatives based on FRET quenching and dequenching of the Biotective Green reagent. The assay requires no wash steps, is amenable to high-throughput platforms, has a rapid readout, and is highly sensitive. It is flexible enough to allow the end-user to either determine the total amount of biotin (via digestion) or effective amount of biotin when attached to a macromolecule. When performed in $0.1 \mathrm{~mL}$, the assay has a lower limit of detection of 4 pmol and a $Z$-factor $>0.9$. 


\section{ACKNOWLEDGMENTS}

The authors kindly thank Jerry Ruth for his insights and discussion, Michael O'Grady and Michael Janes for their careful reading of the manuscript, and Maura Ford for $\alpha$-testing.

\section{COMPETING INTERESTS STATEMENT}

All of the authors are employees of Molecular Probes Detection Technologies, Invitrogen Corporation, the company that sells the Biotective Green reagent.

\section{REFERENCES}

1. Wilchek, M. and E.A. Bayer. 1990. Introduction to avidin-biotin technology, $\mathrm{p}$ 5-13. In M. Wilchek and E.A. Bayer (Eds.), Avidin Biotin Technology: Methods in Enzymology, vol 184. Academic Press, San Diego.

2. Green, N.M. 1965. A spectrophotometric assay for avidin and biotin based on binding of dyes by avidin. Biochem. J. 94:23C-24C.

3. Huang, Z., R.P. Haugland, D. Szalecka, and R.P. Haugland. 1992. A simple and sensitive enzyme-mediated assay of biotin. BioTechniques 13:543-546.

4. Dorris, D.R., R. Ramakrishnan, D. Trakas, F. Dudzik, R. Belval, C. Zhao, A. Nguyen, M. Domanus, and A. Mazumder. 2002. A highly reproducible, linear, and automated sample preparation method for DNA microarrays. Genome Res. 12:976-984.

5. Gan, Z. and R.R. Marquardt. 1999. Colorimetric competitive inhibition method for the quantification of avidin, streptavidin and biotin. J. Biochem. Biophys. Methods 39:1-6

6. Wu, Y., P.C. Simons, G.P. Lopez, L.A. Sklar, and T. Buranda. 2005. Dynamics of fluorescence dequenching of ostrich-quenched fluorescein biotin: A multifunctional quantitative assay for biotin. Anal. Biochem. 342:221228.

7. Pugliese, L., A. Coda, M. Malcovati, and M. Bolognesi. 1993. Three-dimensional structure of the tetragonal crystal form of egg-white avidin in its functional complex with biotin at 2.7 A resolution. J. Mol. Biol. 231:698-710.

8. Livnah, O., E.A. Bayer, M. Wilchek, and J.L. Sussman. 1993. The structure of the complex between avidin and the dye 2-(4'hydroxyazobenzene) benzoic acid (HABA). FEBS Lett. 328:165-168.

9. Rao, S.V., K.W. Anderson, and L.G. Bachas. 1997. Determination of the extent of protein biotinylation by fluorescence binding assay. Bioconjug. Chem. 8:94-98.

10. Lu, J. and R. Zenobi. 1999. Matrix-assisted laser desorption/ionization time-of-flight mass spectrometry for identifying the composition of labeled proteins. Anal. Biochem. 269:312-316.

11.Zhang, J.H., T.D. Chung, and K.R. Oldenburg. 1999. A simple statistical parameter for use in evaluation and validation of high throughput screening assays. J. Biomol. Screen. 4:67-73.

Received 20 June 2007; accepted 2 August 2007.

Address correspondence to Robert $H$. Batchelor, Molecular Probes Detection Technologies, Invitrogen, 29851 Willow Creek Road, Eugene, OR 97402, USA. e-mail: rob.batchelor@invitrogen.com

To purchase reprints of this article, contact: Reprints@BioTechniques.com 